\title{
¿Conocen los profesionales las necesidades de los padres de niños con patología del desarrollo?
}

\section{Do professionals understand the needs of parents of children with developmental disorders?}

\author{
Cordelia Estevez Casellas, Natalia Castello Aracil, Sonia Tirado González \\ Universidad Miguel Hernández, España
}

Disponible online 31 de agosto de 2015

\begin{abstract}
El impacto familiar de los trastornos del desarrollo es una evidencia extensamente descrita que genera intensas respuestas emocionales en los padres y supone un complicado reajuste en las dinámicas familiares. El apoyo emocional y la ayuda psicológica a los progenitores, forma parte del desempeño profesional de los psicólogos que trabajan en el campo de los problemas del desarrollo, ya que esta intervención revertirá en la calidad de atención y en la estabilidad del niño con dificultades. Es fundamental conocer las necesidades de los progenitores y la importancia que les dan a éstas, así como desarrollar instrumentos válidos para los profesionales en la evaluación e intervención de familias. Este trabajo pretende, con un estudio transversal descriptivo en padres, madres y profesionales, describir y comparar estas necesidades. Se encuestaron a 37 padres y madres de niños con patología del desarrollo y a 20 profesionales de diversos centros educativos y de intervención infantil. Los resultados indican que ambos coinciden en el tipo de necesidades que tienen los padres y madres, aunque no en la valoración de la importancia que les dan a éstas.

Palabras Clave: Patología del Desarrollo; Necesidades Familiares; Discapacidad; Impacto Familiar.

Many studies have described the impact on families of children with developmental disorders, which cause a wide range of intense emotional responses in the parents and involve a difficult readjustment period regarding family dynamics. Providing parents with emotional and psychological support forms part of the work of the psychologist who specializes in childhood developmental disorders, because this type of intervention will improve the parents' quality of attention and the stability of the children. Thus, the parent's needs must be understood and the importance that they give to these needs. Valid tools must be developed to guide professionals during the evaluation of these families and interventions. Using a descriptive cross-sectional design that includes parents and professionals, this article describes and compares the needs of this population. A total of 37 parents with children with a developmental disorder were interviewed, as well as 20 professionals from different schools and child intervention centres. The results suggest that both groups agree on the needs of the parents, but not on the importance that they give to them.
\end{abstract}

Key Words: Developmental Pathology; Family Needs; Disabilities; Family Problems.

Correspondencia: Cordelia Estevez Casellas. Departamento Psicologia de la Salud, Universidad Miguel Hernandez. E-mail: c.estevez@umh.es. E-mail de las co-autoras: Natalia Castello: natalia.castello@goumh.umh.es; Sonia Tirado: sonia.tirado@umh.es 
Las dificultades y los problemas del desarrollo en la infancia son centro de numerosas investigaciones, aunque no abundan las centradas en la atención a las familias de estos niños/ as (Romero y Peralta, 2012). A pesar de que en los últimos años ha aumentado el estudio sobre familia y discapacidad (Eckert, 2007), durante muchos años las familias de las personas con discapacidad han sido un colectivo con poca participación a la hora de expresar o plantear sus necesidades (García, 2007).

Todo nacimiento de un niño implica modificaciones en el seno de una familia, pero si el niño nace con alguna Patología del Desarrollo (PD) además hay que añadirle el impacto que supone para la familia la ruptura de las expectativas previas al nacimiento (López, 2011). Algunos autores describen el nacimiento de un hijo con estas características como una respuesta de shock inicial (Davis y Day, 2010; Lozano y Pérez, 2000), que lleva de forma implícita un conjunto de modificaciones y la aparición de necesidades especiales en la familia (López, 2011) produciendo una experiencia prolongada y a veces dolorosa, que puede generar en los padres desajuste psicológico y sufrimiento emocional (De Linares, 1997; Limiñana, Corbalán y Patró, 2007; Suriá, 2011; Wiegner y Donders, 2000), teniendo además en cuenta que esto no se produce sólo al nacer o en el momento de conocer las dificultades del desarrollo, sino que sucede a lo largo de todo el ciclo vital (Giné et al., 2011; Lozano y Pérez, 2000). A pesar de que las familias que tienen un hijo con problemas o riesgo de tenerlos, son familias con necesidades especiales, la realidad es que en su dinámica no tienen por qué ser radicalmente diferentes de las otras familia (De Linares y Rodríguez, 2004).

La familia no sólo cubre las funciones de desarrollo y de socialización en los niños, sino que también cubre la función de desarrollo y de realización personal en sus miembros adultos (Palacios y Rodrigo, 1998) Por tanto, es el contexto socializador por excelencia, donde los miembros que la forman evolucionan y se desarrollan a nivel afectivo, físico, intelectual y social según sus modelos vivenciados e interiorizados (Sarto, 2001). Si hablamos de familia y discapacidad el papel de la familia es aun más relevante ya que las competencias de cada miembro de la familia son recursos importantes que influyen en el modo en que un niño convive con su discapacidad (PereiraSilva y Dessen, 2001) y además el modo en el que la familia convive con él, está relacionado con su desajuste (Robbins, Szapocznik, Tejeda, Samuels, Ironson y Antoni, 2003). Esto hace necesario distanciarse de un enfoque patológico de tipo médico, centrado en la atención de la discapacidad del niño y abrirse hacia una visión contextual y social del sistema familiar en la que emergen nuevas y diferentes aproximaciones a la familia (Córdoba y Soto, 2007).

Esto va en consonancia con el modelo ecológico de Bronfenbrenner (1979) y el transaccional de Sameroff (1987), que constatan la importancia de centrar el trabajo en el contexto familiar, no sólo para intervenir en el desajuste emocional de los padres sino también para la mejora de la calidad de vida del niño con discapacidad.

Es necesario que los profesionales involucrados tengan claro que formar parte de una familia con un miembro con PD no debe ser sinónimo de familia patológica o infeliz. Los conflictos a nivel familiar no surgen como consecuencia directa de la discapacidad, sino en función de las estrategias, recursos y capacidades de la familia para adaptarse a esta situación. Por lo tanto, el trabajo con familias ha de partir de las necesidades que éstas plantean para mejorar su calidad de vida y no de las que los profesionales presuponen (Guevara Benítez y González Soto, 2012) el enfoque centrado en la familia implica la creencia de que los procesos y resultados en el niño se optimizan a través de los servicios que abarcan al niño, la familia y los entornos donde el niño vive, aprende y juega (King et al., 2002; Palisano, Snider y Orlin, 2004).

La realidad, es que se evidencia la importancia de seguir estudiando las necesidades de los padres y las madres de niños con PD y analizar la importancia de cada una de ellas. En esta línea, sería interesante comparar los puntos de vista de los padres y madres con el de los profesionales, sobre las necesidades de estas familias.

Los estudios de Robles y Romero (2011) y De Silva y Elsen (2006) dicen que las familias presentan necesidades diferentes y especiales surgidas del inevitable proceso de adaptación a la situación de discapacidad, demandando un mayor apoyo, cuidado, información, acogida y orientación en las tareas de desarrollo familiar. Por otro lado un estudio interesante de Kreutz y Bosa (2009) centrado en la deficiencia visual, nos dice que los familiares sienten que los profesionales les tratan como si fuesen profesores o terapeutas del niño, con el objetivo constante de enseñarles cómo tratar al niño y descuidando sus necesidades emocionales.

En la bibliografía encontramos estudios realizados desde la atención temprana, como los trabajos de Speck y Peterander (1994), Sarimski (1996), Lanners y Lambert (1999) y Gómez (1999). En resumen, todo ellos indican que las necesidades de las familias se refieren a prevención y diagnóstico precoz, información y formación, atención sanitaria y rehabilitadora, certificado de minusvalía como "estigma", guarderías, centros educativos, recursos económicos, atención psicológica, transporte, ayudas en el hogar, ocio y tiempo libre, contacto con familias y asociaciones, e influencia social.

Por otro lado, desde el ámbito de la educación encontramos los trabajos de Eckert (2002), Thimm y Wachtel (2002), Seifert (2003) y Sodogé y Eckert (2004), que subrayan la importancia que se les da a los niveles de necesidades de los padres y madres, al apoyo personal y familiar y también a las necesidades conyugales.

De manera específica, desde la discapacidad intelectual, están los trabajos de Guillén y Muñoz (2011), que concluyen 
que las necesidades más frecuentes son las referidas a apoyo individualizado, ayudas económicas, asociaciones de familiares y servicios de respiro; y de Giné et al. (2011), que habla de las necesidades de apoyo relacionadas con el acceso a la información, con el bienestar emocional, físico, social, familiar, económico y material; relacionadas con las relaciones con los profesionales y con otras familias y con el impacto de la persona con discapacidad intelectual.

Finalmente, también cabe destacar desde la perspectiva de la enfermedad mental, a Guinea (2007) en su modelo de atención a las personas con enfermedad mental grave, que plantea que las necesidades más frecuentes percibidas por los familiares son las necesidades de información adecuada, de ser tenidos en cuenta en la toma de decisiones que puedan tener repercusiones en la familia, de ayuda material y prestaciones económicas, de tener períodos de descanso o respiro, de apoyo emocional y de apoyo técnico.

Así mismo, también es importante resaltar que FEAPS Madrid (2007) concluye que en la actualidad hay numerosos medios para afrontar las necesidades de las familias de personas con discapacidad intelectual pero que éstos continúan siendo insuficientes.

Esta limitación en la literatura se puede justificar por la escasez que existe de instrumento de evaluación de las necesidades de las madres y padres de niños con PD. Podemos hablar del Family Needs Survey de Bailey y Simeonsson (1988), que tiene seis categorías: a) necesidades de información, b) necesidades de apoyo, c) explicar a los demás, d) servicios comunitarios, e) necesidades financieras, y f) funcionamiento familiar; y el Cuestionario para Valorar las Necesidades de los Padres de Niños con Necesidades Educativas Especiales (CSNP) de Eckert (2009), que tiene cuatro escalas de necesidades: información; asesoramiento; apoyo/alivio; y contactos y comunicación. Como limitaciones de estos dos instrumentos encontramos que no han tenido en cuenta la opinión y el criterio de los propios familiares en su elaboración y no han sido validados en población española. Y, por otro lado, en población española cabe destacar el Cuestionario de Necesidades Familiares de personas con trastornos mentales graves (CNF-TMG) de Gómez, Ruiz, Luz, Freund y Fernández (2012) que agrupa las necesidades en cuatro áreas: conocimientos/información, apoyo instrumental, apoyo personal y participación. Este instrumento tampoco ha tenido en cuenta a los propios familiares durante el proceso de construcción, no tiene la posibilidad de graduar la importancia de cada necesidad y está limitado a los trastornos mentales graves.

Un concepto muy relacionado con las necesidades de estas familias es el de Calidad de Vida Familiar y por tanto cabe destacar dos instrumentos diseñados para evaluar la Calidad de Vida Familiar de las familias que tienen algún miembro con discapacidad y también para planificar la intervención. Estos instrumentos son la Escala de Calidad de Vida Familiar, (Adap- tación de la Family Quality of Life Survey, realizada por el Beach Center on Disability en 2003) de Verdugo, Rodríguez y Sainz (2009) y la Escala de Calidad de Vida (CdVF-E) de Giné et al. (2013).

Dado que los cuestionarios existentes en la actualidad ofrecen poca información sobre la importancia que los padres dan a cada necesidad y los estudios más relevantes sobre el tema tampoco profundizan demasiado en ello, pretendemos, con este estudio describir no sólo las necesidades que surgen en los padres y madres de niños con PD, sino también explorar cuáles son percibidas como las más importantes, tanto desde el punto de vista de los padres y madres como desde el punto de vista de los profesionales. Por lo que los objetivos específicos son:

a) Analizar la presencia o frecuencia de aparición de las diferentes necesidades de madres y padres de niños con PD desde la perspectiva de los propios padres/madres y de los profesionales.

b) Comparar las necesidades descritas por padres/madres y profesionales.

c) Analizar la importancia de cada necesidad desde la perspectiva de los propios padres/madres y de los profesionales.

d) Comparar la importancia de las necesidades entre padres/ madres y profesionales.

\section{Participantes \\ Método}

En el estudio participan dos muestras diferenciadas, una formada por padres/madres y otra por profesionales, ambas no probabilísticas, de conveniencia y voluntarias, siguiendo en ambos casos las normas éticas pertinentes. Los criterios de selección para la muestra de padres/madres son que tengan un hijo con PD, entre 0 y 12 años. Y para la muestra de profesionales que trabajasen en servicios destinados a la intervención de niños con PD.

La muestra de familiares está compuesta por 37 padres de niños con diversos tipo de PD. Concretamente, el 38\% de la muestra son padres y madres que tienen a sus hijos matriculados en el Colegio de Educación Especial Santo Ángel de la Guarda de Alicante, el 32\% en la Asociación Pro-Discapacitados Psíquicos de Alicante (APSA), el 22\% en el centro de Intervención a la Diversidad Educativa y Atención Temprana (IDEAT) y por último el 8\% en el Colegio de Educación Especial Infanta Elena. En total se administraron 65 encuestas, de las cuales se recibieron 40 y 3 de ellas no entraron en el estudio por omisión de respuestas o por respuestas incorrectas, por lo que la tasa de participación fue del $56.90 \%$. Las encuestas fueron contestadas en un $73 \%$ por las madres, en un $19 \%$ por los padres y en un $8 \%$ por ambos. En la Figura 1 se puede apreciar el porcentaje de niños por cada edad, siendo el rango de 1 hasta los 11 años. 
Figura 1

Distribución de los niños por edad.

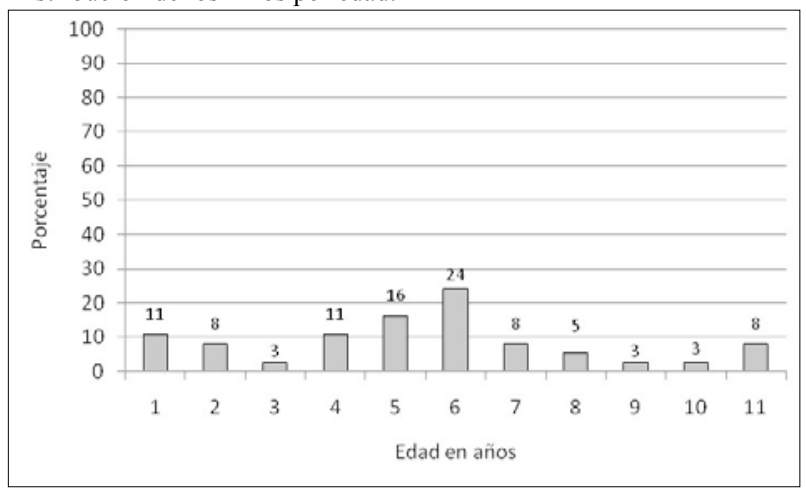

La muestra de profesionales está compuesta por 20 participantes, de los cuales el 50\% son psicólogos, el 35\% maestros, el $5 \%$ educadores, el $5 \%$ fisioterapeutas y el restante $5 \%$ logopedas, procedentes de cuatro centros distintos, de la provincia de Alicante: Colegio de Educación Especial Infanta Elena (30\%), Colegio de Educación Especial Santo Ángel de la Guarda de Alicante (30\%), APSA(20\%) e IDEAT (20\%).Se administraron un total de 26 encuestas, de las cuales se obtuvieron 20 correctamente cumplimentadas, por lo que la tasa de participación fue del $77 \%$.

\section{Variables e instrumentos}

A través de dos encuestas de elaboración propia, una para los profesionales y otra para los padres y madres, se han eva- luado las variables del estudio: presencia e importancia de cada una de las 19 necesidades descritas en la revisión teórica, y que aparecen en la Tabla 1.

La "presencia" de cada necesidad se refiere al hecho de que el padre/madre consideren que han tenido cada necesidad en algún momento; o que el profesional considere que los padres/ madres de niños con PD hayan tenido cada necesidad en algún momento. La variable es dicotómica, siendo las categorías de respuestas SI/NO.

La "importancia" se refiere al grado en que los padres y las madres, y, en el otro caso, los profesionales, consideran que es importante satisfacer cada una de las necesidades. La variable es ordinal, siendo la escala de respuesta tipo Likert de cuatro alternativas $(1=$ muy importante, $2=$ bastante importante, $3=$ algo importante y $4=$ menos importante).

\section{Procedimiento}

Se contactó con la dirección de los centros con el objetivo de obtener los permisos oportunos tanto para acceder a los profesionales como a las familias. Con los consentimientos informados de cada sujeto de la muestra, se procedió a la administración y recogida de las encuestas, que se prolongó desde diciembre del 2012 hasta mayo de 2013. El diseño del estudio es transversal de carácter descriptivo.

Las respuestas se procesaron con el programa IBM ${ }^{\circledR}$ SPSS ${ }^{\circledR}$ v.19 para su posterior análisis estadístico, utilizando las siguientes técnicas: a) análisis de frecuencias para describir el porcentaje de presencia o aparición de cada necesidad en cada

\section{Tabla 1}

Necesidades familiares descritas en la bibliografía.

1) Información. Necesidad de los padres y madres de recibir y comprender cualquier tipo de información relacionada con la situación de tener un hijo con PD (diagnóstico, causa, pronóstico, evolución, tratamientos, estimulación, servicios, etc.).

2) Ayuda Material. Necesidad de los padres y madres de recibir cualquier tipo de ayuda material relacionada con la situación de tener un hijo con PD (sillas especiales, férulas, juegos de estimulación, etc.).

3) Prestaciones Económicas. Necesidad de los padres y madres de recibir prestaciones económicas para solventar todos los gastos derivados de la situación de tener un hijo con PD (gastos de medicamentos, de tratamientos, de cuidadores, etc.).

4) Respiro. Necesidad de los padres y madres de tener períodos de descanso.

5) Apoyo Psicológico. Necesidad de los padres y madres de recibir apoyo emocional o psicológico para poder afrontar la situación de tener un hijo PD.

6) Apoyo Técnico. Necesidad de los padres y madres de formarse en los conocimientos y habilidades necesarios para atender la PD de sus hijos.

7) Toma Decisión. La necesidad de los padres y madres de ser tenidos en cuenta por parte de los profesionales en la toma de decisiones sobre las acciones relacionadas con la PD de sus hijos.

8) Ayuda Hogar. Necesidad de los padres y madres de recibir todo tipo de ayuda en el hogar en relación a la situación de tener que atender a un hijo con PD (ayuda para el cuidado, para la alimentación, etc.).

9) Diagnóstico Precoz. Necesidad de los padres y madres de recibir un diagnóstico más precoz y de recibir indicaciones para prevenir las complicaciones relacionadas con la PD de sus hijos.

10) Certificado Minusvalía. Necesidad de los padres y madres de obtener el certificado de discapacidad oficial que reconoce un porcentaje de minusvalía.

11) Apoyo Escolar. Necesidad de los padres y madres de disponer de más guarderías y centros educativos especializados en PD.

12) Transporte. Necesidad de los padres y madres de recibir cualquier tipo de ayuda relacionada con el transporte diario de los niños con PD.

13) Asociaciones. Necesidad de los padres y madres de tener contacto con otras familias en la misma situación.

14) Atención integral. Necesidad de los padres y madres de que los servicios que atiendan a los niños con PD y los servicios que atiendan a las familias de dichos niños se coordinen y realicen un trabajo global conjunto.

15) Desgaste Físico. Necesidad de los padres y madres de atender el desgaste físico que les sobreviene por la atención de su hijo con PD.

16) Relaciones Familiares. Necesidad de los padres y madres de cuidar y atender las relaciones familiares o los problemas surgidos por la situación de tener un hijo con PD.

17) Relaciones Sociales. Necesidad de los padres y madres de cuidar y atender las relaciones sociales o los problemas surgidos derivados de la situación de tener un hijo con PD.

18) Recursos Humanos. Necesidad de los padres y madres de que hayan más profesionales especializados en las PD.

19) Implicación de los Pediatras. Necesidad de los padres y madres de que los pediatras se impliquen más mejorando su comunicación con ellos durante el tratamiento y en el seguimiento de los niños con PD. 
una de las muestra, b) análisis de tablas de contingencia con la prueba chi-cuadrado para comparar la frecuencia de aparición de cada necesidad en ambas muestras, c) mediana y rango intercuartílico, para describir la importancia de cada necesidad en cada una de las muestras y d) prueba $U$ de Mann-Whitney, para comparar la importancia de cada necesidad en cada muestra. Se estableció un Error tipo I de .01.

\section{Resultados}

Con respecto al objetivo 1 , los datos de la Tabla 2 muestran que, en el caso de los padres/madres, todas las necesidades tienen un porcentaje de aparición superior al 50\%, siendo las más frecuentes Información y Prestaciones Económicas, seguidas de Relaciones Familiares, Apoyo Psicológico, Ayuda Material, Apoyo Técnico, Certificado de Minusvalía, Diagnóstico Precoz, Relaciones Sociales y Recursos Humanos, con un porcentaje superior al $80 \%$. La necesidad menos frecuente ha sido Transporte, con un $54.10 \%$. Por otro lado, en el caso de los profesionales, todas las necesidades tienen un porcentaje de aparición superior al $75 \%$, estando todas sobre el $80 \%$ excepto Desgaste Físico, que ha sido la menos frecuente, y habiendo, además, seis necesidades con un 100\%: Información, Ayuda Material, Prestaciones Económicas, Toma Decisión, Asociaciones y Atención Integral.

Con respecto al objetivo 2, en la Tabla 2 también se puede observar que tan sólo hay diferencias significativas entre padres/madres y profesionales en las necesidades de Asociaciones y Atención Integral. Mientras los padres obtienen un porcentaje medio-alto, del $70 \%$ y del $68 \%$, respectivamente, de aparición de las necesidades, los profesionales obtienen un porcentaje muy alto del $100 \%$ en ambas necesidades.
Tabla 2

Frecuencia de aparición de las necesidades, comparación entre padres y profesionales. Análisis de contingencia.

\begin{tabular}{lcccc}
\hline \multicolumn{1}{c}{ Necesidades } & $\begin{array}{c}\text { Padres } \\
\text { \% aparición }\end{array}$ & $\begin{array}{c}\text { Profesionales } \\
\text { \% aparición }\end{array}$ & $\chi^{2}$ & $p$ \\
\hline Información & 97.30 & 100 & 0.550 & .458 \\
Ayuda Material & 83.80 & 100 & 3.625 & .057 \\
Prest Económicas & 97.30 & 100 & 0.550 & .458 \\
Respiro & 78.40 & 95 & 2.698 & .101 \\
Apoyo Psicológico & 86.50 & 95 & 0.999 & .318 \\
Apoyo Técnico & 83.80 & 95 & 1.516 & .218 \\
Toma Decisión & 78.40 & 100 & 5.030 & .025 \\
Ayuda Hogar & 73.00 & 80 & 0.346 & .556 \\
Diagnóstico Precoz & 81.10 & 85 & 0.138 & .710 \\
Certificado Minusvalía & 83.80 & 90 & 0.416 & .519 \\
Apoyo Escolar & 75.70 & 85 & 0.679 & .410 \\
Transporte & 54.10 & 85 & 5.459 & .019 \\
Asociaciones & 70.30 & 100 & 7.368 & .007 \\
Atención Integral & 67.60 & 100 & 8.216 & .004 \\
Desgaste Físico & 56.80 & 75 & 1.857 & .173 \\
Relaciones Familiares & 89.20 & 95 & 0.548 & .459 \\
Relaciones Sociales & 81.10 & 95 & 2.085 & .149 \\
Recursos Humanos & 81.10 & 85 & 0.138 & .710 \\
Implicación Pediatras & 75.70 & 85 & 0.679 & .410 \\
\hline
\end{tabular}

Con respecto al objetivo 3, los datos de la Tabla 3 muestran cómo en todas las necesidades los padres/madres obtiene un valor central de $M_{e}=1$ (muy importante), excepto en Ayuda Hogar, siendo Información, Apoyo Psicológico, Apoyo Técnico, Diagnóstico Precoz, Apoyo Escolar y Recursos Humanos las necesidades con menor dispersión $(R I Q=0)$. Tanto en Información como en Relaciones Familiares y en Recursos Humanos todos los encuestados han contestado entre 1 y 2 (entre muy importante y bastante importante). Por otro lado, también

Tabla 3

Importancia de las necesidades. Comparación entre padres y profesionales. Prueba $U$ de Mann-Whitney.

\begin{tabular}{|c|c|c|c|c|c|c|c|c|c|c|c|}
\hline \multirow[t]{2}{*}{ Necesidades } & \multicolumn{4}{|c|}{ Padres } & \multicolumn{4}{|c|}{ Profesionales } & \multirow[b]{2}{*}{$U$} & \multirow[b]{2}{*}{$Z$} & \multirow[b]{2}{*}{$p$} \\
\hline & $n^{a}$ & $M_{e}$ & $R I Q$ & Mín.-Máx. & $n^{a}$ & $M_{e}$ & $R I Q$ & Mín.-Máx. & & & \\
\hline Información & 36 & 1.0 & 0 & $1-2$ & 20 & 1 & 1 & $1-4$ & 261 & -2.949 & .003 \\
\hline Ayuda Material & 31 & 1.0 & 1 & $1-3$ & 20 & 2 & 0 & $1-3$ & 184 & -2.663 & .008 \\
\hline Prest Económicas & 36 & 1.0 & 1 & $1-3$ & 20 & 1.50 & 2 & $1-4$ & 269 & -1.768 & .077 \\
\hline Respiro & 29 & 1.0 & 1 & $1-4$ & 19 & 3 & 2 & $1-4$ & 158 & -2.630 & .009 \\
\hline Apoyo Psicológico & 32 & 1.0 & 0 & $1-4$ & 19 & 1 & 2 & $1-4$ & 217 & -2.219 & .026 \\
\hline Toma Decisión & 29 & 1.0 & 1 & $1-3$ & 20 & 2 & 1 & $1-4$ & 113 & -3.938 & .000 \\
\hline Ayuda Hogar & 27 & 2.0 & 1 & $1-4$ & 16 & 2 & 2 & $1-4$ & 118.50 & -2.581 & .010 \\
\hline Diagnóstico Precoz & 30 & 1.0 & 0 & $1-4$ & 17 & 1 & 2 & $1-4$ & 184 & -2.122 & .034 \\
\hline Certificado Minusvalía & 31 & 1.0 & 1 & $1-4$ & 18 & 2.50 & 2 & $1-4$ & 171 & -2.448 & .014 \\
\hline Apoyo Escolar & 28 & 1.0 & 0 & $1-3$ & 17 & 1 & 1 & $1-3$ & 178.50 & -1.799 & .072 \\
\hline Transporte & 20 & 1.0 & 1 & $1-3$ & 17 & 2 & 2 & $1-4$ & 95.50 & -2.468 & .014 \\
\hline Asociaciones & 26 & 1.0 & 1 & $1-3$ & 20 & 2 & 3 & $1-4$ & 152 & -2.551 & .011 \\
\hline Desgaste Físico & 21 & 1.0 & 1 & $1-4$ & 15 & 3 & 2 & $1-4$ & 72.50 & -2.857 & .004 \\
\hline Relaciones Familiares & 33 & 1.0 & 1 & $1-2$ & 19 & 2 & 1 & $1-4$ & 94.50 & -4.569 & .000 \\
\hline Relaciones Sociales & 30 & 1.0 & 1 & $1-3$ & 19 & 3 & 1 & $1-4$ & 126 & -3.451 & .001 \\
\hline Recursos Humanos & 30 & 1.0 & 0 & $1-2$ & 17 & 2 & 2 & $1-4$ & 102.50 & -3.960 & .000 \\
\hline Implicación Pediatras & 28 & 1.0 & 1 & $1-3$ & 17 & 1 & 2 & $1-4$ & 177.50 & -1.699 & .089 \\
\hline
\end{tabular}

Nota. ${ }^{a}$ para cada necesidad hay un número de participantes distinto, que coincide con la frecuencia de aparición. $R I Q:$ rango intercuartílico. $M$ : Mediana. 
podemos apreciar cómo en el caso de los profesionales, tanto Información como Apoyo Escolar son las necesidades más importantes y con menor dispersión $\left(M_{e}=1\right.$ y $\left.R I Q=1\right)$. Aunque con mayor dispersión, también se encuentran entre las más importantes Apoyo Psicológico, Diagnóstico Precoz, Implicación Pediatras y Prestaciones Económicas. Y entre las menos importantes, con una $M_{e}=3$ (algo importante), encontramos Relaciones Sociales, Respiro y Desgaste Físico. Por otra parte, ninguna necesidad tiene una mediana de 4 (menos importante).

En relación al objetivo 4, se observa en la Tabla 3 que, con respecto a la importancia de las necesidades, existen diferencias significativas entre ambas muestras en 11 necesidades: Información, Ayuda Material, Respiro, Apoyo Técnico, Toma Decisión, Ayuda Hogar, Atención Integral, Desgaste Físico, Relaciones Familiares, Relaciones Sociales y Recursos Humanos.

Las Figuras 2, 3, 4, 5, 6 y 7 recogen, para cada necesidad, la distribución de respuesta a cada una de las cuatro categorías de importancia, expresada en porcentajes. Las barras blancas reflejan a los padres/madres, mientras que las grises hacen referencia a los profesionales. Se observa que:

a) En Información, en ambas muestras, el mayor porcentaje de respuestas está en la puntuación 1 (97\% en padres/madres y $70 \%$ en profesionales), pero mientras que en padre/madre el rango de respuesta va de 1 a 2 , en profesionales oscila entre 1 a 4, obteniendo la puntuación 2 también bastantes respuestas en profesionales $(20 \%)$.

b) En Ayuda Material la categoría modal de respuestas en padres/madres está en la puntuación 1 (55\%), seguido de la puntuación $2(35 \%)$, mientras que en profesionales está principalmente en la puntuación $2(65 \%)$.

\section{Figura 2}

Porcentajes de respuestas de Información.

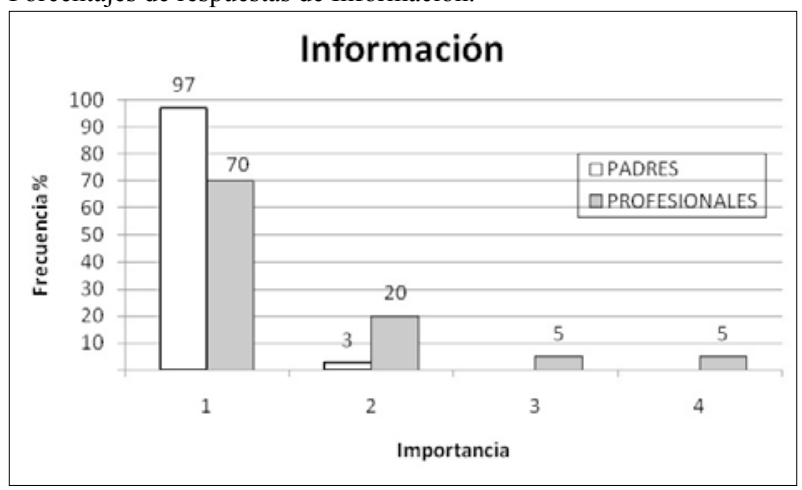

c) En Respiro y en Recursos Humanos destaca en padres/madres la puntuación $1 \quad(55 \%$ para ambas necesidades),mientras que en profesionales las respuestas están repartidas entre todas las puntuaciones, aunque destaca la puntuación 3 (37\% para ambas necesidades).

d) Tanto en Apoyo Técnico como en Toma Decisión, se observa que en padres/madres la puntuación 1 es la que tiene un mayor porcentaje de respuestas ( $81 \%$ y $72 \%$ respectivamente), mientras que en profesionales destaca la puntuación 2 ( $58 \%$ y $40 \%$ respectivamente).

e) También se observa que tanto Ayuda Hogar, como Atención Integral y Desgaste Físico se caracterizan porque las puntuaciones de padres/madres se concentran principalmente en la puntuación 1, seguido de la puntuación $2(48 \%$ y $33,3 \%, 76 \%$ y $20 \%$; y $57 \%$ y $24 \%$ respectivamente) y las puntuaciones de los profesionales se concentran en la puntuación 2, seguido de la 4 (44\% y 25\%, 45\% y 25\%; y $33 \%$ y $33 \%$ ).

f) Por último, se observan diferencias entre las muestras en Relaciones Familiares y Relaciones Sociales, destacando en ambas que, de nuevo, en padres/madres el mayor porcentaje de respuestas está en la puntuación 1 seguido de la puntuación 2 (72\% y 27\% respectivamente en Relaciones Familiares y 53\% y $37 \%$ respectivamente en Relaciones Sociales) y, sin embargo, en la muestra de profesionales las puntuaciones se concentran principalmente en las puntuaciones 2 y 3 , teniendo un porcentaje muy similar en Relaciones Familiares (37\% y 32\% respectivamente) e igual en Relaciones Sociales (32\%).

Figura 3

Porcentajes de respuestas de Ayuda Material.

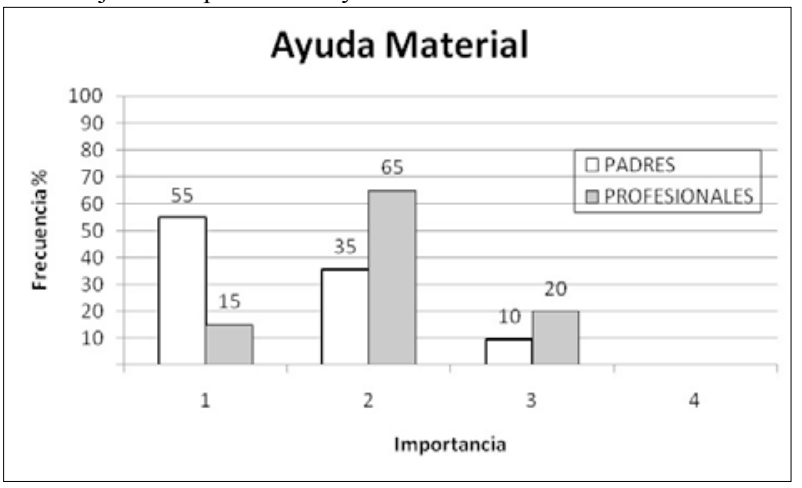


Figura 4

Porcentajes de respuestas de Respiro y Recursos Humanos.

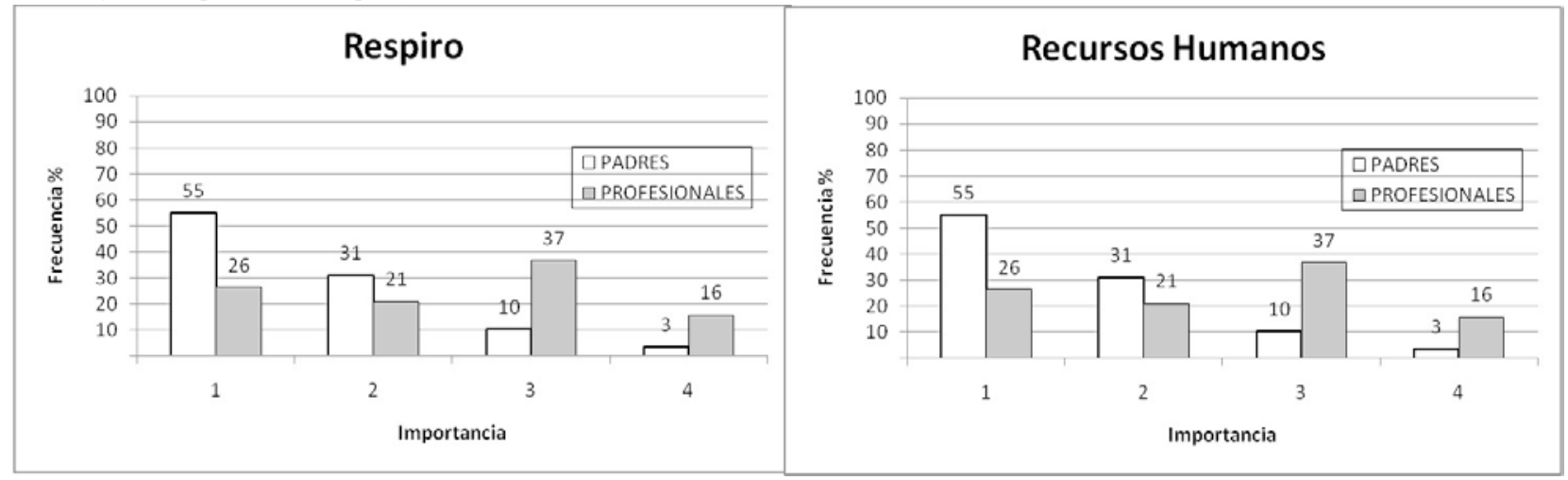

Figura 5

Porcentajes de respuestas de Apoyo Técnico y Toma Decisión.

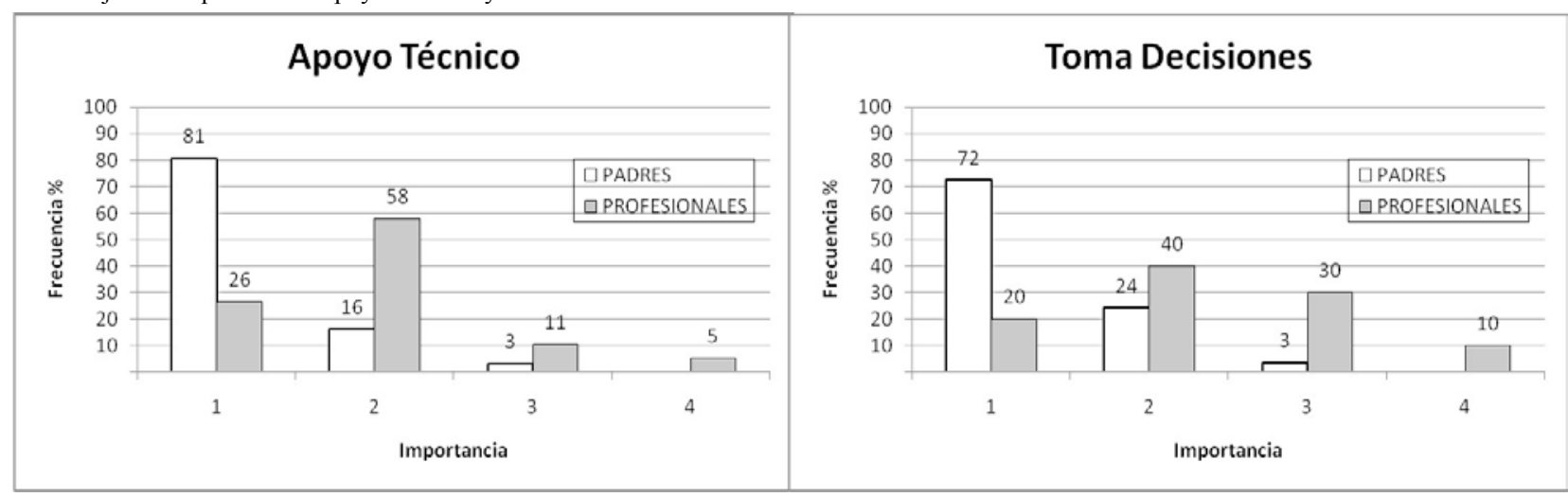

Figura 6

Porcentajes de respuestas de Ayuda Hogar, Atención Integral y Desgaste Físico.
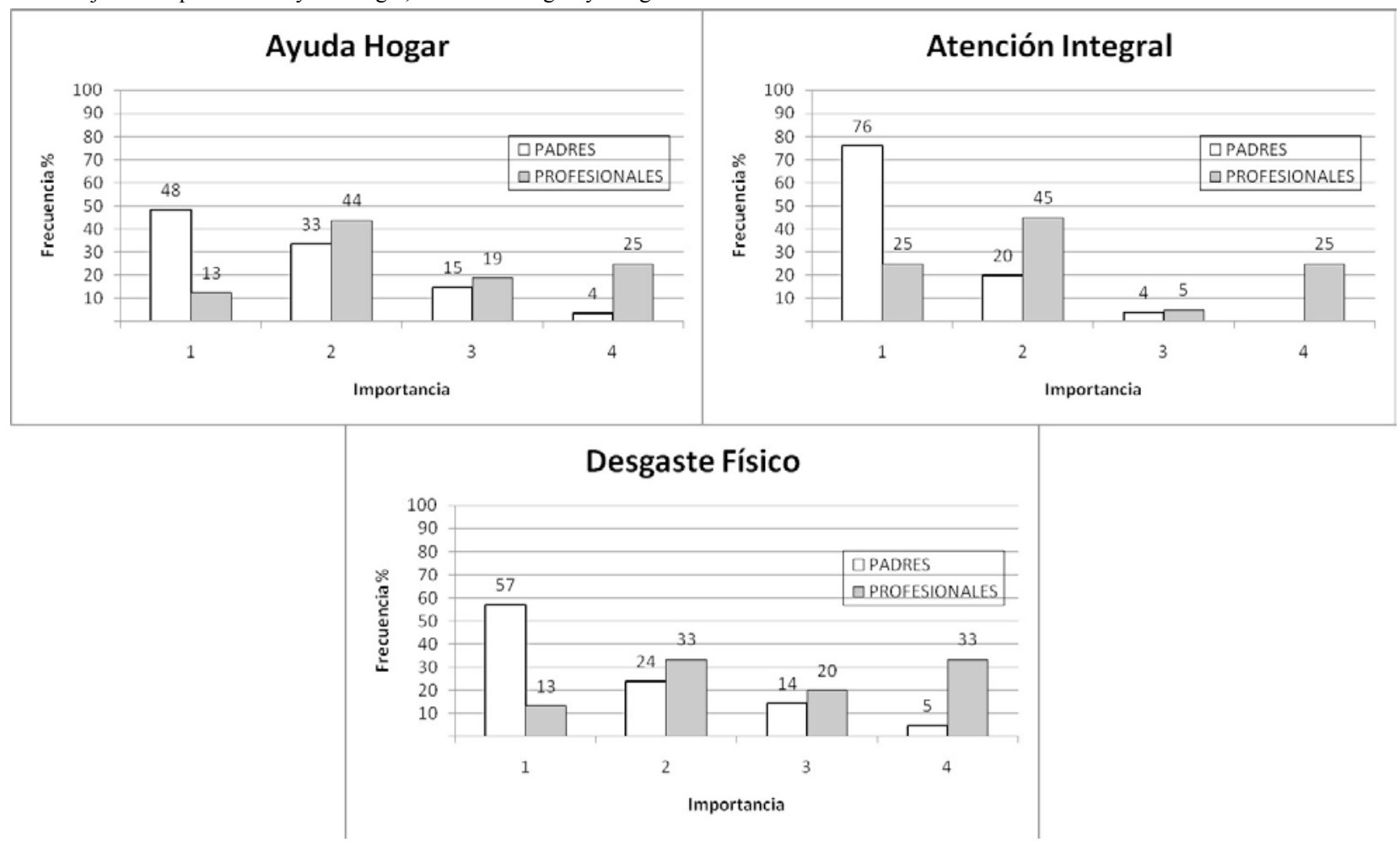
Figura 7

Porcentajes de respuestas de Relaciones Familiares y Relaciones Sociales.

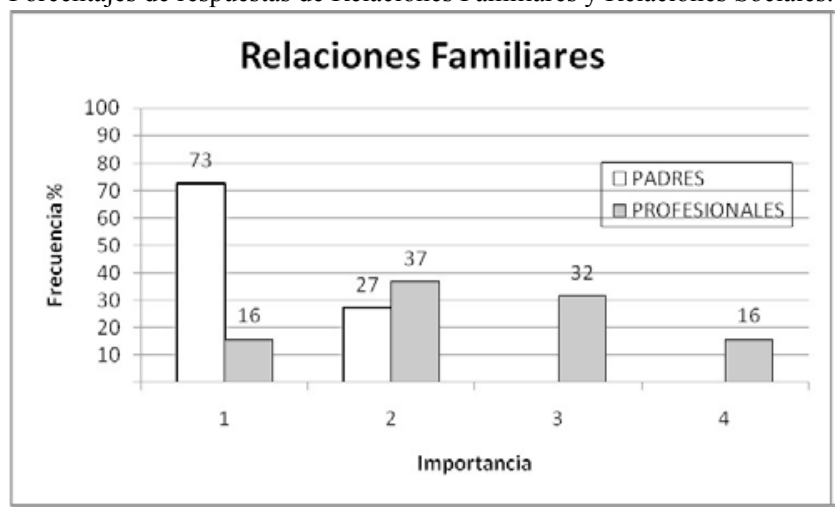

Discusión

Nuestros resultados muestran que las necesidades descritas por nuestra muestra coinciden con las descritas en la bibliografía. No obstante hay que señalar que hay mayor homogeneidad en la presencia de dichas necesidades, entre los profesionales que entre los padres, ya que estos últimos se centran en su propia experiencia. Este resultado es muy valioso, ya que podría sugerir una buena capacidad de los profesionales, para conocer las necesidades generales de las familias con niños con PD.

Por otro lado, nuestro estudio muestra que, en relación a la importancia de cada una de las necesidades, los padres les dan la misma importancia a todas las necesidades, mientras que los profesionales restan la importancia de algunas de ellas, que para los progenitores son de más relevancia. Esto sugeriría la posible tendencia de algunos profesionales a minimizar la magnitud del impacto general de tener un niño con PD.

Concretamente, y en relación a estas diferencias de percepción, hay que señalar que en el caso de la necesidad de información, los padres la perciben como mucho más importante que los profesionales, ya que debido al crecimiento normal del niño, aparecen nuevas dudas en referencia a su problemática y condición y podría ocurrir que los profesionales dieran por hecho que con la información diagnóstica inicial es suficiente.

En lo que se refiere a ayuda material, los padres le dan más importancia que los profesionales, ya que las pequeñas ayudas técnicas facilitan mucho el manejo de padres estos niños para sus pares, y según nuestros datos, para los profesionales es una necesidad de segundo orden.

Es interesante señalar el caso de las necesidades de respiro y de más recursos humanos, que para los profesionales tiene una importancia media-baja y que para los padres es de las más valoradas. Esta discrepancia de percepción puede deberse a la tendencia de los padres a no expresar sus propias necesidades de descanso y de apoyo y a centrar sus demandas en las necesidades de sus hijos.

Algo similar ocurre en referencia a las necesidades de ser partícipes en la toma de decisiones y de recibir formación sobre la problemática de su hijo, nuestros datos informan que para los

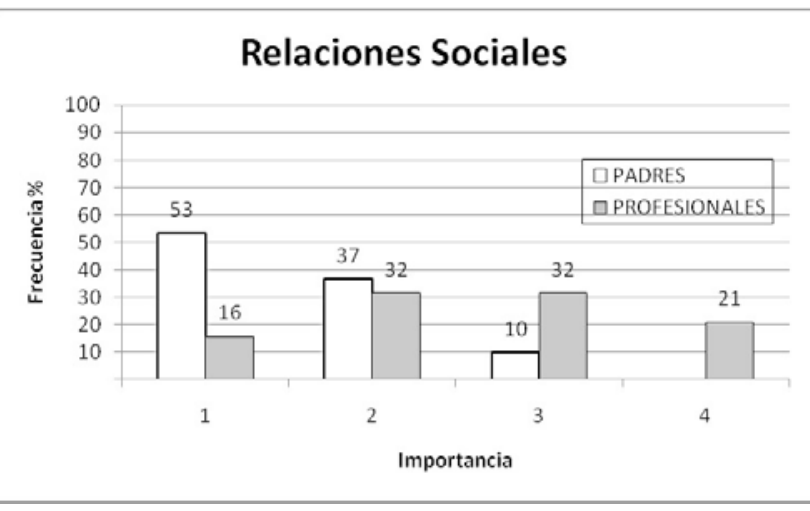

padres es algo más importante que para los profesionales. Esto podría indicar la necesidad de que los padres expresen abiertamente sus necesidades de ser partícipes de manera activa en el proceso de intervención de sus hijos.

$\mathrm{Y}$, en referencia a las necesidades de ayuda en el hogar, de coordinación de una atención integral y de atender su desgaste físico, la diferencia de percepción entre padres y madres y profesionales mantiene las líneas ya descritas, siendo valoradas como más importantes por padres y madres que por profesionales, ya que estos últimos suelen atender más a los aspectos relacionados con la salud del niño y no tanto a las necesidades relacionadas con el manejo cotidiano del niño.

Finalmente, en relación a las necesidades de apoyo social y familiar, nos encontramos de nuevo con la tendencia de los profesionales a darles menos importancia que los propios padres y madres. Esto sugiere la idea ya expresada previamente de la tendencia de los distintos profesionales a prestar menos atención a los aspectos socioafectivos y emocionales de los padres y de las madres de niños con PD, centrándose sobre todo en los aspecto evolutivos, educativos y médicos del niño.

Sobre la base de los resultados encontrados, podemos concluir:

a) Las necesidades de los padres/madres con niños con PD coinciden con las descritas en la bibliografía.

b) La magnitud de la importancia de muchas de las necesidades de los padres/madres de niños con PD en ocasiones son desconocidas por los profesionales.

c) Las necesidades de los padres/madres de niños con PD menos valoradas por los profesionales son aquellas derivadas del manejo diario y la convivencia con un niño de estas características.

d) Es necesario que los profesionales que trabajan en este ámbito conozcan y valoren convenientemente las necesidades de los padres y madres de niños con PD, para darles una respuesta eficaz.

Como líneas de trabajo futuro se hace necesario construir un instrumento que incluya todas las necesidades descritas y las gradúe, permitiendo así al profesional evaluar e intervenir de una manera práctica, operativa y eficaz 
Como limitaciones de nuestro estudio señalamos el rango de edad de los niños de los padres y madres encuestados. Estos niños pertenecen a las etapas de educación infantil y primaria, por lo que los resultados son extrapolables a dichas etapas y sería conveniente ir más allá y estudiar también las etapas de la adolescencia y de la juventud.

\section{Referencias}

1. Bailey, D. B. y Simeonson, R. J. (1988). Assessing needs of families with handicapped infants. Journal of Special Education, 22, 117-127. http://dx.doi. org/10.1177/002246698802200113

2. Bronfenbrenner, U. (1979). The ecology of human development. Cambridge: Harvard University Press.

3. Córdoba, L. y Soto, G. (2007). Familia y discapacidad: intervención en crisis desde el modelo ecológico. Psicología Conductual, 15, 525-541.

4. Davis, H. y Day, C. (2010). Working in Partnership with Parents. London: Pearson.

5. De Linares, C. (1997). Estrés en la familia ante el nacimiento de un hijo con déficil. En M. I. Hombrados (Comp), Estrés y salud (pp. 547-562). Valencia:Promolibro.

6. De Linares, C. y Rodriguez, T. (2004). Bases de la intervención familiar en atención temprana. En J. Pérez-López y A. G. Brito de la Nuez (Coords.), Manual de Atención Temprana (pp. 333-351). Madrid: Piramide.

7. Eckert, A. (2002). Eltern behinderter Kinder und Fachleute. Erfahrungen, Bedürfnisse und chancen. Bad Heilbrunn: Klinkhardt.

8. Eckert, A. (2007). Familien mit einem behinderten Kind - zum aktuellen Stand der wissenschaftlichen Diskussion. Behinderte Menschen, 30, 40-53.

9. Eckert, A. (2009). Diseño y verificación de un instrumento para registrar las necesidades de los padres en cuanto a apoyo personal e institucional. CSNP (cuestionario para valorar las necesidades de los padres de niños con discapacidad). Educatio Siglo XXI, 27, 169-190.

10. Federación de Organizaciones a Favor de Personas con Discapacidad Intelectual Madrid (2007). Necesidades de las Familias de Personas con Discapacidad Intelectual de Feaps Madrid. Madrid: Disponible en: http://www. feapsmadrid.org/documents list?title $=$ Necesidades + de + las +Familias [consulta: 2013, 14 de Mayo]

11. García, J. N. (2007). Dificultades de aprendizaje. Madrid: Pirámide Ediciones S.A.

12. Giné, C., Balcells-Balcells, A.., Simó-Pinatella, D., Font, J., Pro, M. T., Mas, J. M. y Carbó, M. S. (2011). Necesidades de apoyo de las familias de personas con discapacidad intelectual de cataluya. Siglo Cero, 42, 31-49.

13. Giné, C., Vilaseca, R., Gràcia, M., Mora, J., Orcasitas, J.M., Simón, C. y Simó- Pinatella, D. (2013). Spanish Family Quality of Life Scales: Under and over 18years old. Journal of Intellectual \& Developmental Disability, 38, 141-148. doi:10.3109/13668250.2013.774324. http://dx.doi.org/10. 3109/13668250.2013.774324

14. Gómez, E. (1999). Las familias con niños discapacitados. Minusval, 120, 10-11.

15. Gómez, M., Ruiz, A., Luz, M., Freund, N. y Fernández, A. (2012). Construcción y fiabilidad de un cuestionario para evaluar las necesidades familiares de personas con trastorno mental grave. Revista de la Asociación Española de Neuropsiquiatría, 32, 461-479. http://dx.doi.org/10.4321/ $\underline{\mathrm{S} 0211-57352012000300003}$

16. Guevara Benítez, Y. y González Soto, E. (2012). Las familias ante la discapacidad. Revista Electrónica de Psicología Iztacala, 15, 1023-1050.

17. Guillén, A. I. y Muñoz, M. (2011). Variables asociadas a las necesidades psicosociales de personas con enfermedad mental grave usuarias de servicios comunitarios y de sus cuidadores familiares. Anuario de Psicología Clínica y de la salud, 7, 15-24.

18. Guinea, R. (2007). Modelo de atención a las personas con enfermedad mental grave. Madrid: Ministerio de Trabajo y Asuntos Sociales.

19. King, G., Tucker, M. A., Baldwin, P., Lowry, K., LaPorta, J. y Martens, L. (2002) A life needs model of pediatric service delivery: services to support community participation and quality of life for children and youth with disabilities. Physical \& Occupational Therapy in Pediatrics, 22, 53-77. http://dx.doi.org/10.1080/j006v22n02 04

20. Kreutz, C.M. y Bosa, C.A. (2009). Intervenção precoce na comunicação pais-bebê com deficiência visual. Estudos de Psicología, 26, 537-544. http://dx.doi.org/10.1590/S0103166X2009000400013

21. Lanners, R. y Lambert, J. L. (1999). Die Bedürfnisse von Eltern behinderter Kleinkinder. VHN, 68, 36-47.

22. Limiñana, R. M., Corbalán, J. y Patró, R. (2007). Afrontamiento y adaptación psicológica en padre de niños con fisura palatina. Anales de psicología, 23, 201-206.

23. López, C. (2011). El estrés en familias con sujetos con Deficiencia Intelectual. (Tesis Doctoral), Universidad Complutense de Madrid, Madrid.

24. Lozano, M. y Pérez, I. (2000). Necesidades de la familia de las personas con retraso mental y necesidades de apoyo generalizado. En M.A. Verdugo (Ed.), Familias y discapacidad intelectual (pp.199-225). Madrid: FEAPS.

25. Ministerio de Educación, Cultura y Deporte (2012). Datos $y$ cifras. Curso escolar 2012/2012. Madrid: Secretaría General Técnica. Disponible en http://www.mecd.gob.es/ horizontales/estadisticas/indicadores-publicaciones-sintesis/datos-cifras.html [consulta 2013, 7 de mayo]

26. Palacios, J. y Rodrigo, M. J. (1998). La familia como contexto de desarrollo humano. En M. J. Rodrigo y J. Palacios (Coords.), Familia y desarrollo humano (pp. 25-44). Madrid: Alianza.

27. Palisano, R. J., Snider, L. M. y Orlin, M. N. (2004) Recent 
advances in physical and occupational therapy for children with cerebral palsy. Seminars in Pediatric Neurology, 11, 66-77. http://dx.doi.org/10.1016/j.spen.2004.01.010

28. Pereira-Silva, N.L. y Dessen, M.A. (2001). Deficiência mental e familia: Implicações para o desenvolvimento da criança. Psicología: Teoria e Pesquisa, 17, 133-141. http:// dx.doi.org/10.1590/S0102-37722001000200005

29. Robbins, M., Szapocznik, J., Tejeda, M., Samuels, D., Ironson, G. y Antoni, M. (2003). The protective role of the family and social support network in a sample of Hivpositive African American women: Results of a pilot study. Journal of Black Psychology, 29, 17-37. http://dx.doi. org/10.1177/0095798402239227

30. Robles, Z. y Romero, E. (2011). Programas de entrenamiento para padres de niños con problemas de conducta: una revisión de su eficacia. Anales de Psicología, 27, 86-101.

31. Romero, C. y Peralta, S. (2012). Estudio de la dinámica en familias con hijos/as con síndrome de Down. Revista EUREKA, 9, 69-77.

32. Sameroff, A. J. (1987). The social context of development. En N. Eisenberg (Ed.), Contemporary topics in developmental psychology (pp. 273-291). Nueva York: Wiley.

33. Sarimski, K. (1996). Bedürfnisse von Eltern mit behinderten kinder. Frühförderung interdisziplinär, 15, 97-101.

34. Sarto, M. P. (2001). Familia y discapacidad. III Congreso La Atención a la Diversidad en el Sistema Educativo. Universidad de Salamanca. Instituto Universitario de Intelgración en la Colunidad (INICO).
35. Seifert, M. (2003). Zusammenarbeit zwishen Schule und Elternhaus. Geistige Behinderung, 42, 296-310.

36. Silva, A.F. y Elsen, I. (2006). Uma sociedade inclusivista para as famílias por-tadoras de membros com necessidade especial. Família, Saúde e Desenvolvimento, 8, 154-162.

37. Sodogé, A. y Eckert, A. (2004). Kooperation mit Eltern in der Sonderschule - Ergebnisse einer Befragung von Eltern und Sonderschullehrem. Zeitschrift für Heilpädagogik, 10, 453-461.

38. Speck, O. y Peterander, F. (1994). Elternbildung, Autonomie und Kooperation in der Frühförderung. Frühförderung interdisziplinär, 13, 108-120.

39. Suriá Martínez, R. (2011). Discapacidad adquirida y discapacidad sobrevenida: análisis comparativo de la sobrecarga que generan ambas formas de discapacidad en las madres de hijos afectados. Siglo Cero, 42, 67-84.

40. Thimm, W. y Wachtel, G. (2002). Familien mit behinderten kindern. Weinheim: Juventa.

41. Verdugo, M.A., Rodríguez, A. y Sainz, F. (2009). Escala de calidad de vida familiar. Salamanca: INICO.

42. Wiegner, S. y Donders, J. (2000). Predictors of parental distress after con-genital disabilities. Journal Developmental Behavioral Pediatrics, 21, 271-274. http://dx.doi. org/10.1097/00004703-200008000-00003

Fecha de recepción: 20 de enero, 2015 Fecha de recepción de la versión modificada: 5 de mayo, 2015 Fecha de aceptación: 11 de junio, 2015 